\title{
Metabolic syndrome: prevalence and risk factors in Korean gout patients
}

Jae Hyun Jung ${ }^{1}$, Gwan Gyu Song ${ }^{1}$, Jong Dae $\mathrm{Ji}^{2}$, Young Ho Lee ${ }^{2}$, Jae-Hoon Kim ${ }^{1}$, Young Ho Seo ${ }^{3}$, and Sung Jae Choi ${ }^{3}$

${ }^{1}$ Division of Rheumatology, Department of Internal Medicine, Korea University Guro Hospital, Seoul; ${ }^{2}$ Division of Rheumatology, Department of Internal Medicine, Korea University Anam Hospital, Seoul; ${ }^{3}$ Division of Rheumatology, Department of Internal Medicine, Korea University Ansan Hospital, Ansan, Korea

Received: February 26, 2016

Revised : May 13, 2016

Accepted: May 23, 2016

\section{Correspondence to}

Sung Jae Choi, M.D.

Division of Rheumatology,

Department of Internal

Medicine, Korea University

Ansan Hospital, 123 Jeokgeum-ro,

Danwon-gu, Ansan 15355, Korea

Tel: $+82-31-412-6760$

Fax: +82-31-412-5984

E-mail: csjmd888@korea.ac.kr
Background/Aims: We performed this study to investigate associations between metabolic syndrome, chronic kidney disease (CKD), and gout.

Methods: We reviewed the medical records of 151 patients with gout at the Department of Rheumatology in Korea University Ansan Hospital. The following measures were examined: waist circumference, blood pressure, alcohol consumption, and levels of triglyceride, high density lipoprotein cholesterol, fasting serum glucose, serum uric acid (SUA), creatinine, insulin, and C-peptide. We assessed metabolic syndrome by the homeostasis model assessment of insulin resistance (HOMA-IR) index and renal function by the Modification of Diet in Renal Disease equation; patients were classified according to World Health Organization Asia-Pacific obesity criteria.

Results: The prevalence of metabolic syndrome in gout patients (50.8\%) was higher than in non-gout patients. The mean SUA level was significantly higher in gout patients with metabolic syndrome $(9.13 \pm 3.15 \mathrm{mg} / \mathrm{dL})$ than in gout patients without metabolic syndrome $(8.14 \pm 2.07 \mathrm{mg} / \mathrm{dL})$. The mean SUA level was also significantly higher in patients with gout and CKD $(9.55 \pm 2.86 \mathrm{mg} / \mathrm{dL})$ than in patients with gout but no CKD $(7.74 \pm 2.27 \mathrm{mg} / \mathrm{dL})$. In gout patients, HOMA-IR was positively correlated with waist circumference $(r=0.409, p=0.001)$.

Conclusions: The prevalence of metabolic syndrome in patients with gout was $50.8 \%$, which is higher than the prevalence in the general Korean population. Hyperuricemia in gout patients was correlated with metabolic syndrome and CKD. Insulin resistance may provide clues to better understand the relationship between metabolic syndrome, CKD, and gout.

Keywords: Metabolic syndrome; Gout; Renal insufficiency, chronic; Obesity, abdominal; Insulin resistance

\section{INTRODUCTION}

Gout is a disease caused by deposition of monosodium urate monohydrate crystals in joints. Gout is associated with metabolic syndrome related comorbidities such as obesity, hypertension, abnormal lipid metabolism, and impaired glucose tolerance [1]. Previous studies suggest that the prevalence of metabolic syndrome is high among patients with gout, ranging from $30.1 \%$ to $82.0 \%$ [2]. In 2004, Rho et al. [3] showed that the prevalence of metabolic syndrome in Korean gout patients was $59.4 \%$ according to Adult Treatment Panel III (ATP III) criteria with World Health Organization (WHO) Asia-Pacific adjustment; the prevalence was $57.2 \%$ in 2005 [4]. Subsequently, Yoo et 
al. [5] showed that the prevalence was 50.6\% in 2009. A1though the current exact prevalence of metabolic syndrome in Korean gout patients is not known, these results indicate that the prevalence may be decreasing.

The prevalence of metabolic syndrome in the Korean general population in 2012 was $28.2 \%$ according to ATP III criteria with WHO Asia-Pacific adjustment [6]. In Korea, the prevalence of metabolic syndrome has increased in recent years [7] and gout prevalence has also has increased [8]. Thus, in this study we investigated the prevalence of metabolic syndrome in gout patients and determined whether the presence of metabolic syndrome affects serum uric acid (SUA) levels and if the prevalence of metabolic syndrome in Korean gout patients has changed since the last study published 5 years ago.

We also analyzed the relationship between alcohol consumption, chronic kidney disease (CKD), and gout. Multiple studies have shown an association between alcohol intake and hyperuricemia, which is a common precipitating cause of gouty arthritis [9]. Consistent with reports that the prevalence of gout is increasing worldwide, the prevalence of gout in Korea has also been shown to be increasing rapidly. Lee et al. [10] showed that an increase in the frequency and in the amount of alcohol consumption was associated with a higher prevalence of gout. In addition, CKD commonly occurs among gout patients [11]. To date, results from studies that have tested the correlation between metabolic syndrome and CKD $[12,13]$ have reported that patients with greater diagnostic criteria for metabolic syndrome have a higher chance of CKD.

\section{METHODS}

\section{Subjects}

We reviewed the medical records of 151 patients $(138$ men and 13 women) with gout. All patients were seen between 2003 and 2014 at the Department of Rheumatology of Korea University Ansan Hospital. Patients satisfied the preliminary criteria of gout according to guidelines from the American College of Rheumatology [14]. Some patients came to the clinic for acute gout attack and were referred by their employers for the examination. Others were referred by their personal physicians or were self-referred.

\section{Clinical and biochemical investigations}

At clinic visits, the body mass index (BMI) of each patient was calculated using the height and weight. In addition, waist circumference (WC) and blood pressure (BP) at systolic and diastolic phases were measured. Levels of triglycerides (TGs), high density lipoprotein cholesterol (HDL-C), fasting serum glucose, SUA, and creatinine after a 12-hour fast state were measured. In order to study insulin resistance, we measured the concentrations of insulin and C-peptide. Insulin resistance was assessed by the homeostasis model assessment of insulin resistance (HOMA-IR), which is calculated from fasting plasma glucose level and fasting insulin (insulin resistance index) [15]. Renal function in gout patients was evaluated by the estimated glomerular filtration rate (eGFR), calculated using the Modification of Diet in Renal Disease equation [16]. The eGFR was classified as normal, mildly impaired, moderately impaired, or severely impaired according to the following cutoffs: $\geq 90,60$ to 89,30 to 59 , and $<30 \mathrm{~mL} / \mathrm{min} / 1.73 \mathrm{~m}^{2}$, respectively. HOMA-IR and eGFR were calculated using the following equations: HOMA-IR = fasting insulin $(\mu \mathrm{IU} / \mathrm{mL}) \times$ fasting glucose $(\mathrm{mmol} / \mathrm{L}) / 22.5 ; \mathrm{eGFR}=186 \times($ serum creatinine $)-1.154$ $\times$ age -0.203 .

In accordance with a report by the National Cholesterol Education Program, a patient was diagnosed with metabolic syndrome if more than three categories were satisfied among the following five criteria: (1) central obesity (WC greater than $102 \mathrm{~cm}$ in men and $88 \mathrm{~cm}$ in women); (2) concentration of TG greater than $150 \mathrm{mg} / \mathrm{dL}$; (3) concentration of HDL-C less than $40 \mathrm{mg} / \mathrm{dL}$ in men and $50 \mathrm{mg} / \mathrm{dL}$ in women; (4) BP greater than $130 / 85 \mathrm{mmHg}$ or under medical treatment of hypertension; or (5) concentration of serum glucose in fasting state greater than $100 \mathrm{mg} / \mathrm{dL}$ or under medical treatment of diabetes [17]. The first criterion for central obesity was re-evaluated to $90 \mathrm{~cm}$ in men and $80 \mathrm{~cm}$ in women, according to the Asia-Pacific obesity criteria (APC) by the WHO [18]. For patients without WC data, the first criterion for central obesity was substituted with a BMI greater than $25 \mathrm{~kg} / \mathrm{m}^{2}$, in accordance with modified criteria from ATP III [19].

As history of alcohol consumption was not consistently assessed in all patients, we divided patients into two groups: drinking and non-drinking. Patients who drank regularly and consumed more than one bottle a week were included in the drinking group, and the patients 
who drank socially and less than one bottle a week were included in the non-drinking group [10].

\section{Statistical analysis}

Data was analyzed using the software IBM SPSS version 19.0 (IBM Co., Armonk, NY, USA) and results were presented in the form of mean \pm standard deviation. We performed descriptive statistics for general demographic characteristics and calculated the prevalence of metabolic syndrome. Incidence of metabolic syndrome in gout patients was assessed using the chi-square test for categorical data and Student $t$ test for continuous data. We used a multivariate logistic regression model to estimate the odds ratios for clinical and laboratory findings. All confidence intervals were at $95 \%$, and a $p<0.05$ was considered significant.

\section{Etical statement}

This study was performed in accordance with the principles of the Declaration of Helsinki and approved by the Ethics Committee of Korea University Ansan Hospital. The informed consent was waived.

\section{RESULTS}

\section{General characteristics of patients with gout}

A total of 151 patients with gout were included in this study. There were a total of 138 male patients and $13 \mathrm{fe}$ male patients (10.6:1). The mean age of gout onset was $51.28 \pm 15.07$ years, the mean weight was $70.72 \pm 12.99 \mathrm{~kg}$, the mean BMI was $25.07 \pm 3.62 \mathrm{~kg} / \mathrm{m}^{2}$, and the mean WC was $91.86 \pm 9.93 \mathrm{~cm}$. The mean BMI indicated obesity according to APC by the WHO. The mean SUA level was $8.70 \pm 2.64 \mathrm{mg} / \mathrm{dL}$, mean concentration of creatinine was $1.59 \pm 1.15 \mathrm{mg} / \mathrm{dL}$, mean eGFR was $59.08 \pm 19.79 \mathrm{~mL} /$ $\mathrm{min} / 1.73 \mathrm{~m}^{2}$ and mean HOMA-IR was $3.78 \pm 5.93$. A total of $69.5 \%$ of patients had hypertension and $17.9 \%$ had diabetes mellitus (DM). Other general characteristics of study patients are described in Table 1.

\section{The prevalence rate of metabolic syndrome}

Using the WHO APC, we diagnosed 66 of 130 (50.8\%) with metabolic syndrome. Table 2 shows the prevalence of the criteria for metabolic syndrome. Among all patients, $78.8 \%$ had hypertension, $54.7 \%$ had hyperglyceridemia, $46.5 \%$ had low HDL-C, and $33.1 \%$ had high fast-
Table 1. Descriptive characteristics of gout patients

\begin{tabular}{|c|c|}
\hline Factor & Mean \pm SD \\
\hline Age, yr & $51.28 \pm 15.07$ \\
\hline Male & $50.33 \pm 15.11$ \\
\hline Female & $61.38 \pm 10.69$ \\
\hline Height, m & $167.67 \pm 7.34$ \\
\hline Male & $169.04 \pm 5.83$ \\
\hline Female & $153.38 \pm 6.29$ \\
\hline Weight, kg & $70.72 \pm 12.99$ \\
\hline Male & $71.75 \pm 12.96$ \\
\hline Female & $59.92 \pm 7.19$ \\
\hline Body mass index, $\mathrm{kg} / \mathrm{m}^{2}$ & $25.07 \pm 3.62$ \\
\hline Male & $25.03 \pm 3.66$ \\
\hline Female & $25 \cdot 56 \pm 3.39$ \\
\hline Waist circumference, $\mathrm{cm}^{\mathrm{a}}$ & $91.86 \pm 9.93$ \\
\hline Male & $91.45 \pm 10.03$ \\
\hline Female & $96.17 \pm 8.28$ \\
\hline Systolic blood pressure, mmHg & $129.00 \pm 16.31$ \\
\hline Diastolic blood pressure, $\mathrm{mmHg}$ & $81.71 \pm 14.03$ \\
\hline Fasting serum glucose & $108.26 \pm 39.01$ \\
\hline Insulin, $\mu \mathrm{IU} / \mathrm{mL}$ & $13.98 \pm 17.91$ \\
\hline Insulin resistance (HOMA-IR) & $3.78 \pm 5.93$ \\
\hline Total cholesterol, mg/dL & $186.59 \pm 42.86$ \\
\hline Triglyceride, mg/dL & $200.49 \pm 132.76$ \\
\hline HDL-C, mg/dL & $41.87 \pm 14.61$ \\
\hline Uric acid, mg/dL & $8.70 \pm 2.64$ \\
\hline Creatinine, mg/dL & $1.59 \pm 1.15$ \\
\hline $\mathrm{eGFR}, \mathrm{mL} / \mathrm{min} / 1.73 \mathrm{~m}^{2}$ & $59.08 \pm 19.79$ \\
\hline
\end{tabular}

Total number of male patients was 138 and female patients was 13 (10.6:1).

HOMA-IR, homeostasis model assessment of insulin resistance; HDL-C, high density lipoprotein cholesterol; eGFR, estimated glomerular filtration rate.

${ }^{\mathrm{a} O n l y} 69$ values present (males 67, females 2).

ing glucose. Finally, central obesity was diagnosed in $49.3 \%$ (APC) of the patients. The number of normal body weight, overweight, and obese patients was 42 (28.4\%), $44(29.7 \%)$, and 62 (41.9\%), respectively. The prevalence of metabolic syndrome according to age is presented in Table 3.

The average HOMA-IR in gout patients was $3.78 \pm 5.93$. We compared clinical factors between the non-dinking and drinking groups. The mean TG level was higher in 
Table 2. Prevalence rate of each metabolic syndrome criterion in gout patients compared to control groups $(n=150)$

\begin{tabular}{lc}
\hline Variable & No. $(\%)$ \\
\hline Obesity & \\
BMI $>25$ & $148(41.9)$ \\
WC $^{\mathrm{a}}$ & $69(56.5)$ \\
$\mathrm{APC}$ & $150(49 \cdot 3)$ \\
TG $\geq 150$ & $128(54.7)$ \\
HDL-C & $127(46.5)$ \\
Fasting serum glucose $\geq 110$ & $145(33.1)$ \\
BP $\geq 130 / 85$ & $150(78.8)$ \\
\hline
\end{tabular}

BMI, body mass index; WC, waist circumference; APC, Asia-Pacific obesity criteria; TG, triglyceride; HDL-C, high density lipoprotein cholesterol; BP, blood pressure.

${ }^{\mathrm{a}}>90$ Males, $>80$ females.

${ }^{\mathrm{b}}<40$ Males, $<50$ females.
Table 3. The prevalence of metabolic syndrome according to age

\begin{tabular}{lc}
\hline Variable & Metabolic syndrome (APC) \\
\hline Age, yr & \\
$\leq 29$ & $4 / 10(40.0)$ \\
$30-39$ & $9 / 26(34.6)$ \\
$40-49$ & $18 / 38(47 \cdot 4)$ \\
$50-59$ & $14 / 30(46.7)$ \\
$60-69$ & $12 / 24(50.0)$ \\
$70-79$ & $9 / 20(45.0)$ \\
\hline $80-89$ & $0 / 3$ \\
\hline Total & $66 / 151(43.7)$ \\
\hline
\end{tabular}

Values are presented as number (\%). APC, Asia-Pacific obesity criteria.

Table 4. Mean serum uric acid level in gout patients according to metabolic syndrome and chronic kidney disease

\begin{tabular}{|c|c|c|c|}
\hline Variable & No. & SUA, mg/dL & $p$ value \\
\hline \multicolumn{4}{|l|}{ MS } \\
\hline MS+ & 65 & $9.13 \pm 3.15$ & 0.024 \\
\hline MS- & 63 & $8.14 \pm 2.07$ & \\
\hline \multicolumn{4}{|l|}{ CKD } \\
\hline CKD+ & 67 & $9.55 \pm 2.86$ & 0.001 \\
\hline CKD- & 63 & $7.74 \pm 2.27$ & \\
\hline \multicolumn{4}{|l|}{ MS CKD } \\
\hline $\mathrm{MS}+\mathrm{CKD}-^{\mathrm{a}}$ & 31 & $8.15 \pm 2.45$ & 0.161 \\
\hline MS-CKD-b & 32 & $7.34 \pm 2.04$ & \\
\hline $\mathrm{MS}+\mathrm{CKD}^{\mathrm{c}}{ }^{\mathrm{c}}$ & 35 & $10.14 \pm 3.50$ & 0.073 \\
\hline $\mathrm{MS}-\mathrm{CKD}+{ }^{\mathrm{d}}$ & 32 & $8.91 \pm 1.78$ & \\
\hline
\end{tabular}

Values are presented as mean $\pm \mathrm{SD}$.

SUA, serum uric acid; MS, metabolic syndrome; CKD, chronic kidney disease.

${ }^{a}$ With only MS. ${ }^{b}$ Without MS or CKD. ${ }^{c}$ With both metabolic syndrome and CKD. ${ }^{d}$ With only CKD.

the drinking group $(43.22 \pm 11.84 \mathrm{mg} / \mathrm{dL})$ compared to the non-drinking group $(39.83 \pm 16.40 \mathrm{mg} / \mathrm{dL})$; however, this result was not statistically significant, and there were no significant differences between the two groups according to other clinic factors.

\section{Serum uric acid level, chronic kidney disease, and metabolic syndrome}

Table 4 compares SUA levels between gout patients with metabolic syndrome and gout patients without meta- bolic syndrome. We observed that mean SUA levels in gout patients with metabolic syndrome differed significantly from those in gout patients without metabolic syndrome $(9.13 \pm 3.15 \mathrm{mg} / \mathrm{dL}$ vs. $8.14 \pm 2.07 \mathrm{mg} / \mathrm{dL}$, respectively; $p=0.024$ ). We also determined whether SUA levels differed between gout patients with and without CKD. Presence of CKD was defined as having an eGFR less than $60 \mathrm{~mL} / \mathrm{min} / 1.73 \mathrm{~m}^{2}$ [11]. The mean SUA level in patients with gout and CKD was significantly higher than that of patients with gout but no CKD $(9.55 \pm 2.86$ 

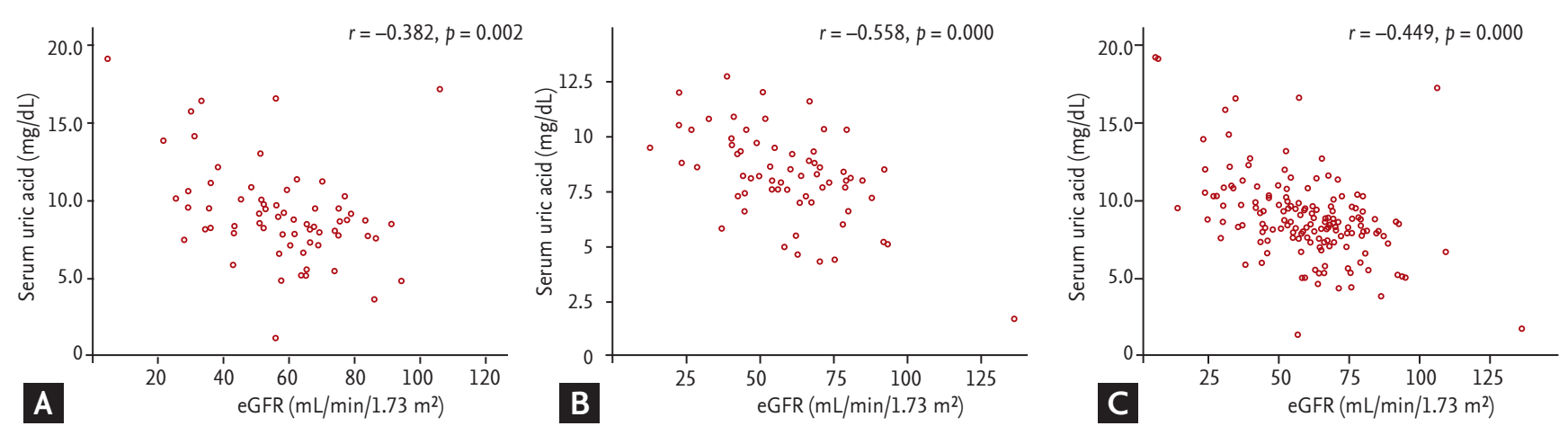

Figure 1. Correlation between serum uric acid level and eGFR among subjects $(A)$ with gout and metabolic syndrome $(n=66)$, (B) with gout and without metabolic syndrome $(n=64)$, and $(C)$ with gout $(n=151)$ by Pearson correlation.

$\mathrm{mg} / \mathrm{dL}$ vs. $7.74 \pm 2.27 \mathrm{mg} / \mathrm{dL}, p=0.001)$.

Based on the results shown in Table 4, we classified patients into four groups: (1) those with both metabolic syndrome and CKD (MS+CKD+); (2) those with only CKD (MS-CKD+); (3) those with only metabolic syndrome (MS+CKD-); and (4) those without metabolic syndrome or CKD (MS-CKD-). We observed differences between the MS+CKD- and MS-CKD- groups, and between the $\mathrm{MS}+\mathrm{CKD}+$ and MS-CKD+ groups; however, these differences were not statistically significant.

We analyzed the correlation between SUA levels and eGFR among gout patients with and without metabolic syndrome. There was a negative correlation between SUA level and eGFR for patients with metabolic syndrome as well as those without metabolic syndrome (Fig. 1). This correlation was stronger in the patients without metabolic syndrome than in patients with metabolic syndrome.

\section{DISCUSSION}

Results from this study showed that the prevalence of metabolic syndrome among Korean gout patients is higher than among the general Korean population and not lower than that of previous studies. Elevated mean SUA levels were found to be significantly increased by the component number of metabolic syndrome [20]; furthermore, the prevalence of metabolic syndrome also increased significantly with SUA level [13]. Although it has been reported that weight loss helps in achieving target SUA level in hyperuricemia or gout patients [21],
Table 5. Correlation between insulin resistance (HOMA-IR) and criterion of metabolic syndrome

\begin{tabular}{lcc}
\hline \multirow{2}{*}{ Variable } & \multicolumn{2}{c}{ HOMA-IR } \\
\cline { 2 - 3 } & $\mathrm{R}$ & $\mathrm{p}$ value \\
\hline Body mass index & 0.317 & 0.000 \\
Waist circumference & 0.409 & 0.001 \\
Systolic blood pressure & 0.078 & 0.392 \\
Diastolic blood pressure & 0.053 & 0.559 \\
Triglyceride & -0.092 & 0.344 \\
HDL-C & 0.014 & 0.890 \\
\hline
\end{tabular}

HOMA-IR, homeostasis model assessment of insulin resistance; HDL-C, high density lipoprotein cholesterol.

weight loss was found to be less effective than medicines that reduced SUA levels. In addition, results from previous studies have suggested that weight loss may prevent the recurrence of acute gout attack [9], weight gain increases the occurrence of gout, and weight loss has a protective effect against gout [22].

Gout is rare in people of younger age and increases in frequency with age, but has recently been observed to be increasingly prevalent in younger adults. The prevalence of gout in adults under 30 years of age was 6.62 $\%$ (10 patients among total 151 patients) in our study. Among these 10 patients, four patients had metabolic syndrome; systolic BP in these patients was greater than $135 \mathrm{mmHg}$. The increasing incidence of gout in younger adults may be related to metabolic syndrome, especially hypertension [1].

The HOMA-IR is a simple method for evaluation of 
insulin sensitivity and is correlated with results of the glucose clamp test in subjects with mild diabetes without significant hyperglycemia [23]. The average HOMAIR in gout patients was $3.78 \pm 5.93$, which was higher than non-gout populations; in a previous study of 7,057 adults, average HOMA-IR was $1.85 \pm 0.96$ [12]. In correlation analyses between HOMA-IR and each diagnostic criterion of metabolic syndrome, BMI and WC showed the most significant positive correlation (Table 5). We concluded that, among the diagnostic criteria for metabolic syndrome, central obesity is most associated with increased insulin resistance in gout patients. As type 2 $\mathrm{DM}$ is associated with insulin sensitivity representing HOMA-IR, future studies should test the correlation between DM and gout.

We investigated the prevalence of metabolic syndrome and CKD. We also observed that patients with metabolic syndrome are more likely to have increased SUA levels, as eGFR is low. In this study, we found that CKD is an independent risk factor for hyperuricemia and gout, and CKD combined with metabolic syndrome was a stronger risk factor. As both metabolic syndrome and CKD are increasing in prevalence in Korea [24,25], prevention of these diseases is important to decrease gout occurrence. Gout is also a risk factor of CKD. In animal studies, injection of hypouricemic agents suppressed the progression of renal disease [26]. Administration of allopurinol to patients with CKD to control hyperuricemia prevents decline of kidney function [27]. For gout patients, allopurinol, febuxostat, or benzbromarone are used as hypouricemic agents.

The strong association between insulin resistance in gout patients and central obesity should be seriously considered. Control of metabolic syndrome is expected to be more useful when medication is limited due to impaired renal function. For gout patients, we suggest combining lifestyle modifications, such as exercise and diet, with medication to reduce risk factors such as occurrence and progression of renal disease, metabolic disease, and cardiovascular disease, which can be achieved with less expense.

This study has several limitations. First, it was a retrospective study conducted at a single center. In addition, the study population was too small for the study to have statistical power. Finally, as gout occurs more frequently in males, the proportion of female patients in this study was small.

In conclusion, the prevalence of metabolic syndrome was higher in patients with gout than in the general Korean population. Hyperuricemia in gout patients has been correlated with metabolic syndrome and CKD, and insulin resistance may provide clues to better understand the relationship between gout, metabolic syndrome, and CKD. Further studies are underway to test the correlation between SUA and kidney function in gout patients under aggressive control of metabolic syndrome. We expect that in gout patients receiving limited medication due to impaired renal function, control of metabolic syndrome will lead to reductions in SUA levels and slow the deterioration of kidney function.

\section{KEY MESSAGE}

1. The prevalence of metabolic syndrome in patients with gout is higher than that in the general Korean population.

2. Hyperuricemia in gout patients is correlated with metabolic syndrome and chronic kidney disease.

3. Average insulin resistance was higher in gout patients, and central obesity had the greatest impact on insulin resistance.

4. Insulin resistance may provide clues to better understand the relationship between gout, metabolic syndrome, and chronic kidney disease.

\section{Conflict of interest}

No potential conflict of interest relevant to this article was reported.

\section{Acknowledgments}

This study was funded by a grant from Korea University Medical College.

\section{REFERENCES}

1. Ichikawa N, Taniguchi A, Urano W, Nakajima A, Yamanaka H. Comorbidities in patients with gout. Nucleosides Nucleotides Nucleic Acids 2011;30:1045-1050.

2. Dao HH, Harun-Or-Rashid M, Sakamoto J. Body composition and metabolic syndrome in patients with primary gout 
in Vietnam. Rheumatology (Oxford) 2010;49:2400-2407.

3. Rho YH, Choi SJ, Lee YH, et al. Prevalence of the metabolic syndrome in patients with gout. J Korean Rheum Assoc 2004;11:349-357.

4. Rho YH, Choi SJ, Lee YH, et al. The prevalence of metabolic syndrome in patients with gout: a multicenter study. J Korean Med Sci 2005;20:1029-1033.

5. Yoo HG, Lee SI, Chae HJ, Park SJ, Lee YC, Yoo WH. Prevalence of insulin resistance and metabolic syndrome in patients with gouty arthritis. Rheumatol Int 2011;31:485-491.

6. Park S, Kim SJ, Lee M, Kang KA, Hendrix E. Prevalence and associated factors of metabolic syndrome among South Korean adults. J Community Health Nurs 2015;32:24-38.

7. Park MY, Kim SH, Cho YJ, Chung RH, Lee KT. Association of leisure time physical activity and metabolic syndrome over 40 years. Korean J Fam Med 2014;35:65-73.

8. Lee CH, Sung NY. The prevalence and features of Korean gout patients using the national health insurance corporation database. J Rheum Dis 2011;18:94-100.

9. Fam AG. Gout, diet, and the insulin resistance syndrome. J Rheumatol 2002;29:1350-1355.

10. Lee CH, Sung NY, Lee J, Bae SC. Factors associated with gout in South Koreans: analysis using the National Health Insurance Corporation and the National Health Screening Exam databases. Clin Rheumatol 2013;32:829837.

11. Jing J, Kielstein JT, Schultheiss UT, et al. Prevalence and correlates of gout in a large cohort of patients with chronic kidney disease: the German Chronic Kidney Disease (GCKD) study. Nephrol Dial Transplant 2015;30:613621.

12. Prasad GV. Metabolic syndrome and chronic kidney disease: current status and future directions. World J Nephrol 2014;3:210-219.

13. Li Y, Chen Y, Liu X, et al. Metabolic syndrome and chronic kidney disease in a Southern Chinese population. Nephrology (Carlton) 2014;19:325-331.

14. Wallace SL, Robinson H, Masi AT, Decker JL, McCarty DJ, Yu TF. Preliminary criteria for the classification of the acute arthritis of primary gout. Arthritis Rheum 1977;20:895-900.

15. Matthews DR, Hosker JP, Rudenski AS, Naylor BA, Treacher DF, Turner RC. Homeostasis model assessment: insulin resistance and beta-cell function from fasting plasma glucose and insulin concentrations in man. Diabetologia
1985;28:412-419.

16. Levey AS, Bosch JP, Lewis JB, Greene T, Rogers N, Roth D. A more accurate method to estimate glomerular filtration rate from serum creatinine: a new prediction equation: Modification of Diet in Renal Disease Study Group. Ann Intern Med 1999;130:461-470.

17. Grundy SM, Cleeman JI, Daniels SR, et al. Diagnosis and management of the metabolic syndrome: an American Heart Association/National Heart, Lung, and Blood Institute scientific statement. Executive Summary. Crit Pathw Cardiol 2005;4:198-203.

18. International Obesity Task Force, World Health Organization Western Pacific Region. The Asia-Pacific perspective: redefining obesity and its treatment 2000. Manila (PH): World Health Organization Western Pacific Region, c2016 [cited 2016 Sep 27]. Available from: http://www.wpro. who.int/nutrition/documents/docs/Redefiningobesity. pdf.

19. Expert Panel on Detection, Evaluation, and Treatment of High Blood Cholesterol in Adults. Executive summary of the third report of the National Cholesterol Education Program (NCEP) expert panel on detection, evaluation, and treatment of high blood cholesterol in adults (Adult Treatment Panel III). JAMA 2001;285:2486-2497.

20. Weiner DE, Tighiouart H, Elsayed EF, Griffith JL, Salem DN, Levey AS. Uric acid and incident kidney disease in the community. J Am Soc Nephrol 2008;19:1204-1211.

21. Zhu Y, Zhang Y, Choi HK. The serum urate-lowering impact of weight loss among men with a high cardiovascular risk profile: the Multiple Risk Factor Intervention Trial. Rheumatology (Oxford) 2010;49:2391239-9.

22. Choi HK, Atkinson K, Karlson EW, Curhan G. Obesity, weight change, hypertension, diuretic use, and risk of gout in men: the health professionals follow-up study. Arch Intern Med 2005;165:742-748.

23. Okita K, Iwahashi H, Kozawa J, et al. Homeostasis model assessment of insulin resistance for evaluating insulin sensitivity in patients with type 2 diabetes on insulin therapy. Endocr J 2013;60:283-290.

24. Kim MK, Lee WY, Kang JH, et al. 2014 Clinical practice guidelines for overweight and obesity in Korea. Endocrinol Metab (Seoul) 2014;29:405-409.

25. Yim HE, Yoo KH. Early life obesity and chronic kidney disease in later life. Pediatr Nephrol 2015;30:1255-1263.

26. Nakagawa T, Mazzali M, Kang DH, et al. Hyperuricemia 
causes glomerular hypertrophy in the rat. Am J Nephrol 2003;23:2-7.

27. Goicoechea M, de Vinuesa SG, Verdalles U, et al. Effect of allopurinol in chronic kidney disease progression and cardiovascular risk. Clin J Am Soc Nephrol 2010;5:13881393 . 